\title{
ON THE EQUIVALENCE OF PROBABILITY SPACES
}

\author{
DANIEL ALPAY, PALLE JORGENSEN, AND DAVID LEVANONY
}

\begin{abstract}
For a general class of Gaussian processes $W$, indexed by a sigma-algebra $\mathscr{F}$ of a general measure space $(M, \mathscr{F}, \sigma)$, we give necessary and sufficient conditions for the validity of a quadratic variation representation for such Gaussian processes, thus recovering $\sigma(A)$, for $A \in \mathscr{F}$, as a quadratic variation of $W$ over $A$. We further provide a harmonic analysis representation for this general class of processes. We apply these two results to: $(i)$ a computation of generalized Ito-integrals; and (ii) a proof of an explicit, and measure-theoretic equivalence formula, realizing an equivalence between the two approaches to Gaussian processes, one where the choice of sample space is the traditional path-space, and the other where it is Schwartz' space of tempered distributions.
\end{abstract}

\section{CONTEnts}

1. Introduction

2. Preliminaries

3. Gaussian processes

3.1. Measure space associated Gaussian processes

3.2. Quadratic variation

3.3. Independent standard Gaussian summands

3.4. Generalized Fourier transform

4. Gaussian stochastic calculus

4.1. Gaussian processes and tempered measures: The Minlos theorem.

4.2. A Gaussian process realized on $\mathcal{S}^{\prime}$.

5. A Gaussian process realized on $C(\mathbb{R})$.

6. Equivalence of measures

6.1. Two probability spaces

6.2. Equivalence

References

\section{INTRODUCTION}

Any stochastic process must be realized in a probability space, a triple made up of a sample space $\Omega$, a sigma-algebra $\mathscr{B}$, and a choice of probability measure $P$ defined on $(\Omega, \mathscr{B})$. In research papers so far, covering a general family of stationary-increment processes, the choice of sample space $\Omega$ has typically been $\mathcal{S}^{\prime}$ (the space of tempered distributions in the sense of Laurent Schwartz). However for applications, a better choice for $\Omega$ (the sample space) is clearly the continuous functions, $C(\mathbb{R})$ if the process is indexed by time (sample paths for

Key words and phrases. .

D. Alpay and P. Jorgensen thank the Binational Science Foundation Grant number 2010117. One of the authors (PJ) thanks colleagues at Ben-Gurion University for kind hospitality, and for many very fruitful discussions. Part of this work was done while PJ visited BGU in May and June 2014. D. Alpay thanks the Earl Katz family for endowing the chair which supported his research. 
the process.) In a setting more general than $\mathcal{S}^{\prime}$ versus $C(\mathbb{R})$ we present an explicit measureisomorphism between the two choices. It will be given by an explicit formula, will be well suited for computations; and our formulas are new even in the case of $\mathcal{S}^{\prime}$ versus $C(\mathbb{R})$. In addition to this isomorphism, we offer a number of applications to stochastic integration.

In our discussion below and in our proofs, we will be making use of the following earlier papers [39, 40, 3. The literature on families of Gaussian processes is vast, both in pure and in applied areas each with a different viewpoint. Our present motivation stems from a certain harmonic analysis, from spectral theoretic computations, and from applications in quantum theory. In our analysis, we study a particular family of Gaussian processes (see sections 2 and 44 and, in our proofs, we utilize of tools from a host of areas. Specifically, we utilize families of operators in Hilbert spaces, with emphasis on explicit reproducing kernel Hilbert spaces. To refer the reader to useful references, we mention the following papers/books whose theme is close to that adopted here: [2, 3, 5, 21, 17, 29, 30, 48, and for papers/books stressing the Hilbert space, see e.g., [25, 44, 10, 22, 26, 15, 42, 4, 38, 34, 43, and the papers cited therein. We make use of basic tools from harmonic analysis, and Gaussian processes in Hilbert space; for background references, see e.g., [20, 31, 32, 8, 7, 6].

The paper is organized as follows: In the first half of the paper (sections 24), we introduce a general class of Gaussian processes indexed by a sigma-algebra of a general but fixed measure space. This material will aid us in two ways, in the second half of the paper, sections 5.6. First it will unify our approach to generalized Ito-integrals, subsequently studied. Secondly, our general theory will be used in the proofs of our results covering our main theme, i.e., setting up a measure-theoretic equivalence between the two approaches to the formulation of Gaussian processes outlined above. This thread culminates with Theorem 6.4.

\section{Preliminaries}

Below we briefly sketch the framework for the particular class of Gaussian processes to be studied in detail. We first gather some notations and definitions needed in the sequel. We already mentioned the Schwartz space $\mathcal{S}$ and its dual $\mathcal{S}^{\prime}$. The duality between the two spaces will be denoted as

$$
\langle\xi, \varphi\rangle, \varphi \in \mathcal{S} \text { and } \xi \in \mathcal{S}^{\prime} \text {, the distribution } \xi \text { applied to } \varphi .
$$

Definition 2.1. Let $M=\mathbb{R}$ and let $\mathscr{F}=\mathscr{B}(\mathbb{R})$ denote the Borel sigma-algebra. A measure $\sigma$ on $\mathscr{B}(\mathbb{R})$ is called tempered if it satisfies

$$
\int_{\mathbb{R}} \frac{d \sigma(u)}{\left(u^{2}+1\right)^{p}}<\infty
$$

for some $p \in \mathbb{N}_{0}$.

The measure $\sigma$ will be called symmetric if

$$
\sigma(A)=\sigma(-A), \quad \forall A \in \mathscr{B} .
$$

Definition 2.2. A cylinder set in $\mathcal{S}^{\prime}$ is a subset of $\mathcal{S}^{\prime}$ of the form

$$
\left\{\xi \in \mathcal{S}^{\prime} ;\left(\left\langle\xi, \varphi_{1}\right\rangle, \ldots,\left\langle\xi, \varphi_{n}\right\rangle\right) \in A\right\}
$$

where $\varphi_{1}, \ldots, \varphi_{n}$ are preassigned in the $S$ chwartz space $\mathcal{S}$, and $A \subset \mathbb{R}^{n}$ is an open subset of $\mathbb{R}^{n}$.

We denote the Fourier transform by

$$
\widehat{f}(\lambda)=\int_{\mathbb{R}} e^{-i \lambda x} f(x) d x, \quad \text { for } \quad f \in \mathbf{L}_{2}(\mathbb{R}) .
$$


Theorem 2.3. Let $\sigma$ be fixed. There is a real-valued Gaussian process $X^{(\sigma)}$ indexed by the space of Schwartz functions $\mathcal{S}$ and realized on a probability space $\left(\mathcal{S}^{\prime}, \mathscr{C}, P^{(\sigma)}\right)$, where $\mathscr{C}$ denotes the cylinder algebra, such that, with $\mathbb{E}^{(\sigma)}[u]=\int_{\mathcal{S}^{\prime}} u d P^{(\sigma)}$ and with the notation (2.1),

$$
X_{\varphi}^{(\sigma)}(\xi)=\langle\xi, \varphi\rangle
$$

we have

and

$$
\mathbb{E}^{(\sigma)}\left[X_{\varphi}^{(\sigma)}\right]=0, \quad \forall \varphi \in \mathcal{S}
$$

We will return to a detailed construction of this process in Sections 446, but we first need some technical preparation.

Proof of Theorem 2.3. In the construction of the Gaussian process

$$
\left(X_{\varphi}^{(\sigma)}\right)_{\varphi \in \mathcal{S}} \quad \text { on } \quad\left(\mathcal{S}^{\prime}, \mathscr{C}, P^{(\sigma)}\right)
$$

in Theorem 2.3, we "invert" a transform. Specifically, we consider the right hand side in (2.6) as a continuous and positive definite function on $\mathcal{S}$, and we apply Minlos' theorem to verify the existence of the Gaussian process (2.7).

Remark 2.4. By this approach, we do not get explicit formulas for (2.7) that can be computed on sample-paths. This situation is remedied in Theorems 5.1 and 6.4 below; see especially formulas (5.3) $-(5.6)$.

\section{Gaussian Processes}

This section is divided into four parts. In the first, our starting point is a given sigma-finite measure space $(M, \mathscr{F}, \sigma)$, and we outline properties of a Gaussian process $W^{(\sigma)}$ which is indexed canonically by the sets from the sigma-algebra of $M$. In subsection 3.2 , we prove an inversion-formula: We show that if $\sigma$ is refinable (Definition 3.20), then this measure $\sigma$ may be recovered as a quadratic variation computed from $W^{(\sigma)}$. Moreover, with this, we formulate and prove a generalized Ito-lemma for $W^{(\sigma)}$. And, as a result, we obtain a general stochastic integration. We use this in the subsequent Section 3.3 to introduce a coordinate system (Theorem 3.34) on the probability space $\Omega$ carrying the process. Moreover, we obtain (Corollary 3.38) a generalized Fourier transform in $\mathbf{L}_{2}(\Omega, P)$. We write the proofs in the case of real valued functions, and real valued random variables, but the arguments apply mutatis mutandis for the complex case as well.

\subsection{Measure space associated Gaussian processes.}

Definition 3.1. Let $(M, \mathscr{F}, \sigma)$ be a sigma-finite measure space, meaning that there exists an increasing sequence $A_{1} \subset A_{2} \subset \cdots$ of elements of $\mathscr{F}$, such that $\cup_{n=1}^{\infty} A_{n}=M$ and $\sigma\left(A_{n}\right)<\infty$ for all $n \in \mathbb{N}$. We say that the Gaussian process $W=\left(W_{A}^{(\sigma)}\right)_{A \in \mathscr{F}}$ is associated with $(M, \mathscr{F}, \sigma)$ if there is a probability space $(\Omega, \mathcal{B}, P)$ such that the following two conditions hold:

(i) Setting $\mathbb{E}[X]=\int_{\Omega} X(\omega) d P(\omega)$, we have

$$
\begin{aligned}
\mathbb{E}\left[W_{A}\right] & =0, \quad \forall A \in \mathscr{F}, \\
\mathbb{E}\left[W_{A} W_{B}\right] & =\sigma(A \cap B), \quad \forall A, B \in \mathscr{F} .
\end{aligned}
$$


(ii) For every finite sequence $A_{1}, A_{2}, \ldots, A_{n}$ of elements of $\mathscr{F}$, the collection of random variables

$$
W_{A_{1}}, W_{A_{2}}, \ldots, W_{A_{n}}
$$

is jointly Gaussian with covariance

$$
\mathbb{E}\left[W_{A_{i}} W_{A_{j}}\right]=\sigma\left(A_{i} \cap A_{j}\right), \quad i, j=1, \ldots, n .
$$

Lemma 3.2. For every sigma-finite measure space $(M, \mathscr{F}, \sigma)$, an associated Gaussian process exists.

Proof. The function $K(A, B)=\sigma(A \cap B)$ is positive definite on $\mathscr{F}$; and so it is the covariance function of a zero-mean Gaussian process with covariance function $\sigma(A \cap B)$, as follows from [33. pp. 466-467].

We note that in the present setting, reference [39] is also helpful for a general discussion of positive definite kernels and Gaussian processes.

Example 3.3. The Cameron-Martin space revisited: Let $(M, \mathscr{F}, \sigma)=\left(\mathbb{R}_{+}, \mathscr{B}, \lambda\right)$, where $\mathbb{R}_{+}$ is the half-line $[0, \infty), \mathscr{B}$ denotes the Borel subsets, and $\lambda$ is the restriction of the Lebesgue measure to $[0, \infty)$. Then the reproducing kernel Hilbert space associated with the positive definite function

$$
K(A, B)=\lambda(A \cap B), \quad A, B \in \mathscr{B},
$$

is the Cameron-Martin Hilbert space $\mathcal{H}_{1}^{(\lambda)}$ where

$$
\mathcal{H}_{1}^{(\lambda)}=\left\{f \in \mathbf{L}_{2}\left(\mathbb{R}_{+}\right) \mid f^{\prime} \in \mathbf{L}_{2}\left(\mathbb{R}_{+}\right) \text {, and } f(0)=0\right\}
$$

and

$$
\|f\|_{\mathcal{H}_{1}^{(\lambda)}}^{2}=\int_{0}^{1}\left|f^{\prime}(x)\right|^{2} d x, \quad f \in \mathcal{H}_{1}^{(\lambda)} .
$$

Discussion of Example 3.3: For $s, t \in[0, \infty)$ set $A=[0, s]$ and $B=[0, t]$. Then

$$
\lambda(A \cap B)=s \wedge t=k(s, t)=k_{s}(t),
$$

and

$$
\left\langle f, k_{s}\right\rangle_{\mathcal{H}_{1}^{(\lambda)}}=f(s), \quad \forall f \in \mathcal{H}_{1}^{(\lambda)} \quad \text { and } \quad s \in[0, \infty)
$$

Remark 3.4. The conclusion in the example above is still valid if the measure $\sigma$ for $\left(\mathbb{R}_{+}, \mathscr{B}, \sigma\right)$ is tempered; see Definition 2.1. The modifications are as follows:

(i) With $\sigma$ given, we get $W^{(\sigma)}$ and its covariance kernel. The corresponding reproducing kernel Hilbert space $R K(\sigma)$ is described as follows:

$$
R K(\sigma)=\left\{F: F(t)=\int_{0}^{t} f(x) d \sigma(x) \text { for some } f \in \mathbf{L}_{2}(\sigma)\right\}
$$

with norm

$$
\|F\|_{R K(\sigma)}^{2}=\int_{0}^{\infty}|f(x)|^{2} d \sigma(x)
$$

and

(ii) the reproducing kernel for $R K(\sigma)$ is

$$
k^{(\sigma)}(t, s)=\sigma([0, t \wedge s]) .
$$


Lemma 3.5. If $(M, \mathscr{F}, \sigma)$ is a sigma-finite measure space, and $\left(W_{A}^{(\sigma)}\right)_{A \in \mathscr{F}}$ is an associated Gaussian process on the probability space $(\Omega, \mathcal{B}, P)$, then the Ito integral

$$
\int_{M} f(x) d W^{(\sigma)}(x) \in \mathbf{L}_{2}(\Omega, \mathcal{B}, P)
$$

exits for all $f \in \mathbf{L}_{2}(M, \mathscr{F}, \sigma)$, and the isometry

$$
\mathbf{E}\left[\left|\int_{M} f(x) d W^{(\sigma)}(x)\right|^{2}\right]=\int_{M}|f(x)|^{2} d \sigma(x)
$$

holds.

Proof. Let $f_{s}$ be a generic simple function, that is

$$
f_{s}(x)=\sum_{k=1}^{n} c_{k} \chi_{A_{k}}(x), \quad n \in \mathbb{N}, \quad A_{1}, \ldots, A_{n} \in \mathscr{F} \quad \text { and } \quad c_{1}, \ldots, c_{n} \in \mathbb{R}
$$

where $\chi_{A_{k}}$ denotes the appropriate indicator function with the sets $A_{1}, \ldots, A_{n}$ being such that $A_{j} \cap A_{k}=\emptyset$ for $j \neq k$, and $c_{1}, \ldots, c_{n} \in \mathbb{R}$. Set

$$
\int_{M} f_{s}(x) d W^{(\sigma)}(x)=\sum_{k=1}^{n} c_{k} W_{A_{k}}^{(\sigma)}
$$

We claim that (3.4) holds. The desired conclusion (3.4) follows for all $f \in \mathbf{L}_{2}(M, \mathscr{F}, \sigma)$ as the simple functions are dense in this latter space and since (3.4) is a densely defined isometry between Hilbert spaces, and thus has a unique everywhere and continuous isometric extension. Indeed (and with the limit meaning approximation by simple functions),

$$
\begin{aligned}
\mathbb{E}\left[\left|\int_{M} f(x) d W^{(\sigma)}(x)\right|^{2}\right) & =\lim \sum_{j, k=1}^{n} c_{j} c_{k} \mathbb{E}\left[W_{A_{j}}^{(\sigma)} W_{A_{k}}^{(\sigma)}\right] \\
& =\lim \sum_{j, k=1}^{n} c_{j} c_{k} \sigma\left(A_{j} \cap A_{k}\right) \\
& =\lim \sum_{k=1}^{n} c_{k}^{2} \sigma\left(A_{k}\right) \\
& =\int_{M}|f(x)|^{2} d \sigma(x)
\end{aligned}
$$

as claimed.

Remark 3.6. A refinement of the proof of Lemma 3.5, for deterministic functions, also yields the case of Ito-integral and Ito-isometry, for stochastic adapted process. The purpose of the extension is to set the stage for the Ito lemma (Corollary 3.25) where the reasoning relies on the generality of stochastic adapted processes, indexed by a given measure space. But for pedagogical reasons, we have chosen to first state the result in the easier special case of the Wiener integration for deterministic functions. Below we now point out how the proof of this case carries over, with suitable modifications, to the case of Ito-integration, and we establish the Ito-isometry for the case of a family of processes which we call $\mathcal{F}$-adapted. See Definition 3.23 and Lemma 3.24 .

Corollary 3.7. Let $\left(W_{A}^{(\sigma)}\right)_{A \in \mathscr{F}}$ be a Gaussian process defined on the probability space $(\Omega, \mathscr{B}, P)$, and let $I=(a, b]$ be a finite interval. Take an $A \in \mathscr{F}$ such that $0<\sigma(A)<\infty$. 
Then

$$
P\left(\left\{\omega \in \Omega \mid W_{A}^{(\sigma)}(\omega) \in I\right\}\right)=P\left(\left\{a<W_{A}^{(\sigma)} \leq b\right\}\right)=\frac{1}{\sqrt{2 \pi}} \int_{\frac{a}{\sqrt{\sigma(A)}}}^{\frac{b}{\sqrt{\sigma(A)}}} e^{-\frac{x^{2}}{2}} d x
$$

Corollary 3.8. Let $W^{(\sigma)}(f), f \in \mathbf{L}_{2}(\sigma)$ be as in (3.7). Then,

$$
\mathbb{E}_{P_{\sigma}}\left[W^{(\sigma)}\left(f_{1}\right) W^{(\sigma)}\left(f_{2}\right)\right]=\left\langle f_{1}, f_{2}\right\rangle_{\sigma}\left(=\int_{M} f_{1}(u) f_{2}(u) d \sigma(u)\right), \quad \forall f_{1}, f_{2} \in \mathbf{L}_{2}(\sigma) .
$$

Proof. This is the polarization of

$$
\mathbb{E}_{P_{\sigma}}\left[\left(W^{(\sigma)}(f)\right)^{2}\right]=\|f\|_{\sigma}^{2}
$$

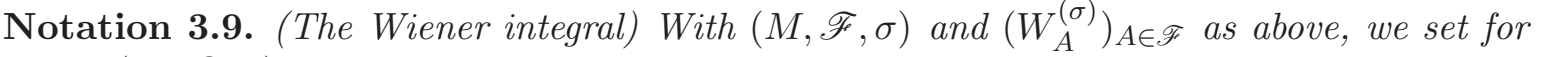
$f \in \mathbf{L}_{2}(M, \mathscr{F}, \sigma)$

$$
W^{(\sigma)}(f)=\int_{M} f d W^{(\sigma)}
$$

Let $(M, \mathscr{F}, \sigma)$ be as above with $W^{(\sigma)}$ being the associated Gaussian process. Let $H_{0}, H_{1}, \ldots$ denote the Hermite polynomials, defined by the generating function

$$
e^{z x-\frac{z^{2}}{2}}=\sum_{n=0}^{\infty} \frac{z^{n}}{n !} H_{n}(x)
$$

Let $\psi \in \mathcal{S}$ be a (real-valued) Schwartz function, with Hermite expansion

$$
\psi(x)=\sum_{n=0}^{\infty} c_{n} H_{n}(x), \quad x \in \mathbb{R}
$$

and set

$$
[\psi](x) \stackrel{\text { def. }}{=} \sum_{n=0}^{\infty} n ! c_{n}^{2} x^{n}, \quad x \in \mathbb{R}
$$

With these notations we can now state:

Corollary 3.10. With $W^{(\sigma)}, \psi$ and $[\psi]$ as above we have the following: Let $f_{1}, f_{2} \neq 0$ be in $\mathbf{L}_{2}(\sigma)$. Then,

$$
\mathbb{E}_{P_{\sigma}}\left[\psi\left(\frac{1}{\left\|f_{1}\right\|_{\sigma}} W^{(\sigma)}\left(f_{1}\right)\right)\left(\psi\left(\frac{1}{\left\|f_{2}\right\|_{\sigma}} W^{(\sigma)}\left(f_{2}\right)\right)\right)\right]=[\psi]\left(\frac{\left\langle f_{1}, f_{2}\right\rangle_{\sigma}}{\left\|f_{1}\right\|_{\sigma} \|_{f_{2} \|_{\sigma}}}\right) .
$$

Proof. By Lemma 3.5 applied to the $\mathcal{N}(0,1)$ random variables $\frac{1}{\left\|f_{1}\right\|_{\sigma}} W^{(\sigma)}\left(f_{1}\right)$ and $\frac{1}{\left\|f_{2}\right\|_{\sigma}} W^{(\sigma)}\left(f_{2}\right)$ we have

$$
\mathbb{E}_{P_{\sigma}}\left[\left(H_{n}\left(\frac{1}{\left\|f_{1}\right\|_{\sigma}} W^{(\sigma)}\left(f_{1}\right)\right)\right)\left(H_{k}\left(\frac{1}{\left\|f_{2}\right\|_{\sigma}} W^{(\sigma)}\left(f_{2}\right)\right)\right)\right]=n ! \delta_{k, n}\left(\frac{\left\langle f_{1}, f_{2}\right\rangle_{\sigma}}{\left\|f_{1}\right\|_{\sigma} \|_{f_{2} \|_{\sigma}}}\right)^{n}
$$

Consider now the left hand side of (3.11), and replace $\psi$ by its expansion (3.9). Taking into account (3.12) we get the right hand side of (3.11).

In the computation of (3.12), we use the following fact about Gaussian vectors in $\mathbb{R}^{2}$. 
Lemma 3.11. Let $c \in \mathbb{R},|c|<1$, and let $\gamma_{2}^{(c)}$ be the $\mathbb{R}^{2}$-Gaussian joint density with covariance matrix $\left(\begin{array}{ll}1 & c \\ c & 1\end{array}\right)$, then for the Hermite functions $H_{n}$ and $H_{k}$ we have:

$$
\iint_{\mathbb{R}^{2}} H_{n}(x) H_{k}(y) d \gamma_{2}^{(c)}(x, y)=\delta_{n, k} n ! c^{n}, \quad n, k \in \mathbb{N}_{0} .
$$

The proof is by direct computation, making use of the Ornstein-Uhlenbeck semigroup $P_{t}$ given by

$$
\left(P_{t} h\right)(x)=\int_{\mathbb{R}} h\left(e^{-t} x+\sqrt{1-e^{-2 t}} y\right) d \gamma_{1}(y), \quad h \in \mathbf{L}_{2}\left(\mathbb{R}, d \gamma_{1}\right) .
$$

For details see for instance [37, pp. 9-28].

Corollary 3.12. Let $W^{(\sigma)}, \psi$ and $[\psi]$ as above, and let $A, B \in \mathscr{F}$ be such that $0<\sigma(A)<\infty$ and $0<\sigma(B)<\infty$. Then,

$$
\mathbb{E}_{P_{\sigma}}\left[\left(\psi\left(\frac{1}{\sqrt{\sigma(A)}} W_{A}^{(\sigma)}\right)\right)\left(\psi\left(\frac{1}{\sqrt{\sigma(B)}} W_{B}^{(\sigma)}\right)\right)\right]=[\psi]\left(\frac{\sigma(A \cap B)}{\sqrt{\sigma(A) \sigma(B)}}\right) .
$$

Definition 3.13. Let $(M, \mathscr{F}, \sigma)$ and $\left(W_{A}^{(\sigma)}\right)_{A \in \mathscr{F}}$ be as in Definition 3.1. We say that a (finite or) countable family $A_{1}, A_{2}, \ldots \in \mathscr{F}$ is a partition of $A$ if $A=\cup_{k \in \mathbb{N}} A_{k}$, and $A_{k} \cap A_{n}=\emptyset$ for $k \neq n$.

Corollary 3.14. Let $\sigma$ be a tempered Borel measure on $\mathbb{R}$, and let $\left(X_{\varphi}^{(\sigma)}\right)_{\varphi \in \mathcal{S}}$ be the corresponding Gaussian process, namely $X_{\varphi}^{(\sigma)}(\xi)=\langle\xi, \varphi\rangle$. Then,

$$
X_{\varphi}^{(\sigma)}=\int_{\mathbb{R}} \widehat{\varphi}(u) d W_{u}^{(\sigma)}
$$

where $W^{(\sigma)}$ in (3.16) is the process in Definition [3.1, and the integral is the generalized Ito integral of Lemma 3.5 .

Proof. Pick a finite partition $\left\{x_{j}\right\}$ of $\mathbb{R}$ such that the integral in (3.16) is approximated by

$$
\sum_{j} \widehat{\varphi}\left(x_{j}\right) W_{\left[x_{j}, x_{j+1}\right)}^{(\sigma)} .
$$

The approximation is in the $L_{2}$ norm in $\mathbf{L}_{2}\left(\mathcal{S}^{\prime}, P_{\sigma}\right)$. Using

$$
\prod_{j} \mathbb{E}_{P_{\sigma}}\left[e^{\left.i \widehat{\varphi}\left(x_{j}\right) W_{\left[x_{j}, x_{j}+1\right.}^{(\sigma)}\right)}\right]=e^{-\frac{1}{2} \sum_{j}\left|\widehat{\varphi}\left(x_{j}\right)\right|^{2} \sigma\left(\left[x_{j}, x_{j+1}\right)\right.},
$$

we conclude, upon passing to the limit of refinements, that

$$
\mathbb{E}_{\sigma}\left[e^{i X_{\varphi}^{(\sigma)}}\right]=e^{-\frac{1}{2} \int_{\mathbb{R}}|\widehat{\varphi}(u)|^{2} d \sigma(u)}
$$

Equivalently, the Gaussian process defined in (3.16), satisfies equation (2) of Theorem 2.3 . By Minlos' theorem, the process in (2) is uniquely determined by the characteristic function $e^{-\frac{1}{2} \int_{\mathbb{R}}|\widehat{\varphi}(u)|^{2} d \sigma(u)}$. 


\subsection{Quadratic variation.}

Lemma 3.15. Let $(M, \mathscr{F}, \sigma)$ be a measure space with $\sigma$ being sigma-finite, and let $\left(W_{A}^{(\sigma)}\right)_{A \in \mathscr{F}}$ be the Gaussian process of Definition 3.1. Then for its moments we have:

(1) The odd moments vanish,

$$
E_{P_{\sigma}}\left[\left(W_{A}^{(\sigma)}\right)^{2 k+1}\right]=0, \quad k \in \mathbb{N}_{0}
$$

and

(2) the even moments are given by:

$$
E_{P_{\sigma}}\left[\left(W_{A}^{(\sigma)}\right)^{2 k}\right]=(2 k-1) ! !(\sigma(A))^{k}, \quad k \in \mathbb{N},
$$

for all $A \in \mathscr{F}$ such that $\sigma(A)<\infty$ where,

$$
(2 k-1) ! !=\frac{(2 k) !}{2^{k} k !} .
$$

We note that in particular we have:

$$
\begin{aligned}
\mathbb{E}_{P_{\sigma}}\left[W_{A}^{(\sigma)}\right] & =0, \\
\mathbb{E}_{P_{\sigma}}\left[\left(W_{A}^{(\sigma)}\right)^{2}\right] & =\sigma(A), \\
\mathbb{E}_{P_{\sigma}}\left[\left(W_{A}^{(\sigma)}\right)^{4}\right] & =3(\sigma(A))^{2} .
\end{aligned}
$$

Proof of Lemma 3.15. The asserted moment expressions in the lemma follow from comparing powers in the moment generating function

$$
\mathbb{E}_{P_{\sigma}}\left[e^{i t W_{A}^{(\sigma)}}\right]=e^{-\frac{t^{2} \sigma(A)}{2}},
$$

valid for all $A \in \mathscr{F}$ such that $\sigma(A)<\infty$.

Proposition 3.16. Let the process $\left(W_{A}^{(\sigma)}\right)_{A \in \mathscr{F}}$ be realized on $\mathbf{L}_{2}(\Omega, \mathscr{B}, P)$ as outlined in Lemma 3.5, and let $\mathbb{E}$ be the expectation operator defined by $P$. Then for any $A \in \mathscr{F}$ and every partition $\left(A_{k}\right)_{k \in \mathbb{N}}$ of $A$, the following identity holds:

$$
\mathbb{E}\left[\left|\sigma(A)-\sum_{k}\left(W_{A_{k}}^{(\sigma)}\right)^{2}\right|^{2}\right]=2 \sum_{k}\left(\sigma\left(A_{k}\right)\right)^{2}=2 \sum_{k}\left(\mathbb{E}\left[\left(W_{A_{k}}^{(\sigma)}\right)^{2}\right]\right)^{2}
$$

Proof. We compute the left hand side in (3.18) with the use of Lemma 3.5 as follows: We first note that the random variables $W_{A_{k}}^{(\sigma)}$ and $W_{A_{n}}^{(\sigma)}$ are independent when $k \neq n$. This follows from (3.3) together with the fact that they are Gaussian; see Corollary 3.7. Set $s=\sigma(A)$ and $s_{k}=\sigma\left(A_{k}\right)$ (so that $s=\sum_{k=1}^{\infty} s_{k}$ ). When $k \neq n$ we have (using independence)

$$
\mathbb{E}\left[\left(W_{A_{k}}^{(\sigma)}\right)^{2}\left(W_{A_{n}}^{(\sigma)}\right)^{2}\right]=s_{k} s_{n}
$$

This is utilized so as to compute the right hand side of (3.18). We get

$$
\begin{aligned}
\mathbb{E}\left[\left|\sigma(A)-\sum_{k=1}^{\infty}\left(W_{A_{k}}^{(\sigma)}\right)^{2}\right|^{2}\right] & =s^{2}-2 s^{2}+\sum_{k=1}^{\infty} \mathbb{E}\left[\left(W_{A_{k}}^{(\sigma)}\right)^{4}\right]+2 \sum_{k<n} s_{k} s_{n} \\
& =-s^{2}+3 \sum_{k=1}^{\infty} s_{k}^{2}+2 \sum_{k<n} s_{k} s_{n} \\
& =2 \sum_{k=1}^{\infty} s_{k}^{2},
\end{aligned}
$$


which is the desired right hand side of (3.18).

Definition 3.17. Let $(M, \mathscr{F}, \sigma)$ and $\left(W_{A}^{(\sigma)}\right)_{A \in \mathscr{F}}$ be as above. For $A \in \mathscr{F}$, we denote by $\operatorname{PAR}(A)$ the set of all $\mathscr{F}$-partition of $A$, and we denote by

$$
\operatorname{Var}_{-}^{(\sigma)}(A)=\inf _{\left(A_{k}\right)_{k \in \mathbb{N}} \in \operatorname{PAR}(A)} \sum_{k=1}^{\infty}\left(\sigma\left(A_{k}\right)\right)^{2}
$$

the lower variation of sum of squares.

Corollary 3.18. Let $A \in \mathscr{F}$, and consider the sum of random variables squares

$$
\sum_{k=1}^{\infty}\left(W_{A_{k}}^{(\sigma)}\right)^{2}
$$

on $\Omega$ for all partitions of $A$. It is $\chi^{2}$-distributed on the probability space $(\Omega, \mathscr{B}, P)$ of Lemma 3.5. Then the following two conditions are equivalent. Given $\varepsilon>0$, and

$$
\left(A_{k}\right) \in \operatorname{PAR}(A) \text { satisfies } \operatorname{Var}_{-}^{(\sigma)}(A)=0 \text { and } \sum_{k}\left(\sigma\left(A_{k}\right)\right)^{2} \leq \epsilon,
$$

$$
\mathbb{E}\left[\left|\sigma(A)-\sum_{k}\left(W_{A_{k}}^{(\sigma)}\right)^{2}\right|^{2}\right] \leq 2 \varepsilon .
$$

\section{Remark 3.19.}

(a) The meaning of $(i i)$ is the assertion that, when $\operatorname{Var}_{-}^{(\sigma)}=0$, the random variable

$$
\sum_{k=1}^{\infty}\left(W_{A_{k}}^{(\sigma)}\right)^{2}
$$

is constant $P$-a.e. on $\Omega$. (It is called the quadratic variation of $W_{A}^{(\sigma)}$ ).

(b) The conditions in the Corollary are satisfied if $(M, \mathscr{F}, \sigma)$ is taken to be the real line with the Borel sets and the Lebesgue measure. For example if $A=[0, T]$ we consider a sequence of partitions consisting of dyadic intervals and

$$
\operatorname{Var}_{-}^{(\sigma)}([0, T]) \leq T^{2} \sum_{k=1}^{2^{n}}\left(\frac{1}{2^{n}}\right)^{2}=\frac{T^{2}}{2^{n}} \rightarrow 0
$$

as $n \rightarrow \infty$.

(c) More generally, consider $(M, \mathscr{F}, \sigma)$ as above, and let $A \in \mathscr{F}$ be such that $0<\sigma(A)<\infty$. Suppose that for all $n \in \mathbb{N}$ there is a partition $\left\{A_{1}, \ldots, A_{n}\right\}$ such that

$$
\sigma\left(A_{k}\right)=\frac{1}{n} \sigma(A), \quad k=1, \ldots, n .
$$

Then

$$
\operatorname{Var}_{-}^{(\sigma)}(A)=0
$$

Indeed, if (3.20) holds, then

$$
\sum_{k=1}^{n} \sigma\left(A_{k}\right)^{2}=\frac{\sigma(A)^{2}}{n} \rightarrow 0
$$

so (3.21) follows.

Definition 3.20. Let $(M, \mathscr{F}, \sigma)$ be specified as above. We say that it is refinable if for every $A \in \mathscr{F}$ and every $\varepsilon>0$ there exists $\left(A_{k}\right) \in \operatorname{PAR}(A)$ such that

$$
\left|\left(A_{k}\right)\right|=\sup _{k} \sigma\left(A_{k}\right)<\varepsilon .
$$


Corollary 3.21. Let $(M, \mathscr{F}, \sigma)$ and $\left(W_{A}^{(\sigma)}\right)_{A \in \mathscr{F}}$ be as above, and assume that $(M, \mathscr{F}, \sigma)$ is refinable. Then for every $A \in \mathscr{F}$ and every $\left(A_{k}^{(n)}\right) \in \operatorname{PAR}(A)$ such that

$$
\lim _{n \rightarrow \infty}\left|A_{k}^{(n)}\right|=0
$$

we have

$$
\lim _{n \rightarrow \infty} \sum_{k}\left(W_{A_{k}^{(n)}}^{(\sigma)}\right)^{2}=\sigma(A), \quad \text { P a.e. }
$$

namely, in the limit, the left hand side random variable reduces to the constant $\sigma(A)$.

Proof. Using Proposition 3.16 and Corollary 3.18 we only need to show that

$$
\lim _{n \rightarrow \infty} \sum_{k}\left(\sigma\left(A_{k}^{(n)}\right)\right)^{2}=0 .
$$

But the following holds for the left hand side of (3.24):

$$
\sum_{k}\left(\sigma\left(A_{k}^{(n)}\right)\right)^{2} \leq\left|A_{k}^{(n)}\right| \sum_{k} \sigma\left(A_{k}^{(n)}\right) .
$$

We now use that, by assumption,

$$
\lim _{n \rightarrow \infty}\left|A_{k}^{(n)}\right|=0
$$

and that $\sum_{k} \sigma\left(A_{k}^{(n)}\right)=\sigma(A)<\infty$ since $\left(A_{k}^{(n)}\right) \in \operatorname{PAR}(A)$ for all $n \in \mathbb{N}$. The conclusion (3.24) now follows.

Remark 3.22. It is obvious that if $(M, \mathscr{F}, \sigma)$ is refinable, it has to be non-atomic.

Definition 3.23. The stochastic process $Y$ defined on $M$ with values in $\mathbf{L}_{2}(\Omega, \mathcal{B}, P)$ is called $\mathcal{F}$-adapted if the following condition holds for every $A, B \in \mathcal{F}$ be such that $A \cap B=\emptyset$. Set $\mathcal{F}_{B}$ to be the sigma-algebra generated by the random variables $W_{C}^{(\sigma)}$, where $C$ runs through all subsets of $B$ which belong to $\mathcal{F}$. For all $x \in A, Y_{x}$ is $\mathcal{F}_{A}$-measurable, and, in addition, is independent of $\mathcal{F}_{B}$.

Lemma 3.24. Let $Y$ be a $\mathcal{F}$-adapted process such that the function $x \mapsto E\left(|Y(x)|^{2}\right)$ is measurable and $\int_{M} \mathbb{E}\left(|Y(x)|^{2}\right) d \sigma(x)<\infty$. Then the random variables

$$
\sum_{k=1}^{n} Y\left(x_{k}\right) W_{A_{k}}^{(\sigma)}
$$

where $\left\{A_{k}\right\}_{k=1}^{n}$ is a covering of $M$ by pairwise disjoint measurable sets and $x_{k} \in A_{k}, k=$ $1, \ldots, n$, converges to an element in $\mathbf{L}_{2}(\Omega, \mathcal{B}, P)$, which we denote $\int_{M} Y(x) d W_{x}^{(\sigma)}$. Furthermore, we have the Ito-isometry property

$$
\mathbb{E}\left(\left|\int_{M} Y(x) d W_{x}^{(\sigma)}\right|^{2}\right)=\int_{M} \mathbb{E}\left(|Y(x)|^{2}\right) d \sigma(x) .
$$

Proof. When computing the difference between two such sums, say $\left\{A_{k}\right\}_{k=1}^{n}$ (with points $\left\{x_{k}\right\}_{k=1}^{n}$ ) and $\left\{B_{k}\right\}_{k=1}^{m}$ (with points $\left\{y_{k}\right\}_{k=1}^{m}$ ) we build a covering of pairwise disjoint measurable sets from the two given covering, say $\left\{C_{k}\right\}_{k=1}^{p}$ (with points $\left\{z_{k}\right\}_{k=1}^{p}$ ) The integral

$$
\mathbb{E}\left(\left|\sum_{k=1}^{n} Y\left(x_{k}\right) W_{A_{k}}^{(\sigma)}-\sum_{k=1}^{m} Y\left(y_{k}\right) W_{B_{k}}^{(\sigma)}\right|^{2}\right)
$$


can then be divided into two groups of terms: The sum

$$
\mathbb{E}\left(\mid \sum_{k=1}^{m}\left(Y\left(z_{i_{k}}\right)-\left.Y\left(z_{j_{k}}\right) W_{C_{k}}^{(\sigma)}\right|^{2}\right)=\sum_{k=1}^{p} \mathbb{E}\left(\left|Y\left(z_{i_{k}}\right)-Y\left(z_{j_{k}}\right)\right|^{2}\right) \sigma\left(C_{k}\right)\right.
$$

which goes to 0 since $\int_{M}\left(\mathbb{E}\left(|Y(x)|^{2}\right)\right) d \sigma(x)<\infty$ and the cross-products

$$
\sum_{k, \ell=1}^{p} \mathbb{E}\left(Y\left(z_{k}\right) W_{C_{k}}^{(\sigma)} \overline{Y\left(z_{\ell}\right)} W_{C_{\ell}}^{(\sigma)}\right)=\left|\mathbb{E}\left(\sum_{k=1}^{p} Y\left(z_{k}\right) W_{C_{k}}^{(\sigma)}\right)\right|^{2}
$$

which goes to 0 since $\sigma$ is refinable, and hence non-atomic.

Corollary 3.25. Let $(M, \mathscr{F}, \sigma)$ be a sigma-finite measure space and assume it is refinable (see Definition [3.20). Let $\left(W_{A}^{(\sigma)}\right)_{A \in \mathscr{F}}$ be the corresponding Gaussian process (see Definition 3.1). Let $f: \mathbb{R} \longrightarrow \mathbb{R}$ be a given $C^{2}$-function. Then for all $A \in \mathscr{F}$ such that $0<\sigma(A)<\infty$ we have:

$$
f\left(W_{A}^{(\sigma)}\right)-f(0)=\int_{A} f^{\prime}\left(W_{x}^{(\sigma)}\right) d W_{x}^{(\sigma)}+\frac{1}{2} \int_{A} f^{\prime \prime}\left(W_{x}^{(\sigma)}\right) d \sigma(x) .
$$

A sketch of the proof. First note that all the terms in (3.26) are random variables. Given

$W_{A}^{(\sigma)}$, by $f\left(W_{A}^{(\sigma)}\right)$ we mean the composition of the function $W_{A}^{(\sigma)}$ from $\Omega$ to $\mathbb{R}$ with $f$, and similarly for the terms under the integrals on the right hand side of (3.26). Further, we stress that the first term $\int_{A} f^{\prime}\left(W_{x}^{(\sigma)}\right) d W_{x}^{(\sigma)}$ is an Ito integral in the sense of Lemma 3.24 and Remark 3.19, but now with the random process $f^{\prime}\left(W_{x}^{(\sigma)}\right)$ occuring under the integral. By the arguments of Lemma 3.5, we have

$$
\mathbb{E}_{P_{\sigma}}\left[\left|\int_{A} f^{\prime}\left(W_{x}^{(\sigma)}\right) d W_{x}^{(\sigma)}\right|^{2}\right]=\int_{A} \mathbb{E}_{P_{\sigma}}\left[\left|f^{\prime}\left(W_{x}^{(\sigma)}\right)\right|^{2}\right] d \sigma(x) .
$$

With that we note that the proof of $(3.26)$ is concluded through the use of the same arguments utilized in the proof of the classical Ito formula, see e.g. [41]. Specifically, these include $(i)$ integration by parts; (ii) a stopping/truncation argument, enabling to prove the result for $W_{A}$ restricted to compacts; (iii) then, on compacts, any $C^{2}$ function may be written as a limit of polynomial functions; and, finally, $(i v)$ the use of standard convergence together with Proposition 3.16 and a probabilistic Dominated Convergence allows to complete the proof.

Remark 3.26. We refer to [3, Theorem 8.2] for a Ito formula where the stochastic term is computed as a Wick product integral, in the setting of an associated Gelfand triple.

Remark 3.27. We note that Ito integration is done with stochastic integrands, say $Z_{t}$, being adapted with respect to an underlying filtration, namely, an increasing sequence of sub-sigma algebras $\mathcal{F}_{t}$. This is to say that for all $t \geq 0, Z_{t}$ is $\mathcal{F}_{t}$-measurable. Given the fact that no natural order may be invoked in the present general setting, such terminology obviously becomes irrelevant here. This is where Definition 3.23 is called for.

\subsection{Independent standard Gaussian summands.}

Example 3.28. Take $M=\mathbb{R}$ and let $\mathscr{F}=\mathscr{B}(\mathbb{R})$ denote the Borel sigma-algebra. Let $\sigma$ be a tempered measure, that is, subject to (2.2), so that we have the Gelfand triple with continuous inclusions

$$
\mathcal{S} \hookrightarrow \mathbf{L}_{2}(\sigma) \hookrightarrow \mathcal{S}^{\prime}
$$


where $\mathcal{S}$ denotes the Schwartz functions and $\mathcal{S}^{\prime}$ denotes the tempered Schwartz distributions. Let $\mathscr{C}$ be the $\mathcal{S}-\mathcal{S}^{\prime}$ cylinder sigma-algebra of subsets of $\mathcal{S}^{\prime}$. We then take

$$
(\Omega, \mathscr{C})=\left(\mathcal{S}^{\prime}, \mathscr{C}\right)
$$

and we note that the corresponding Gaussian process $\left(W^{(\sigma)}\right)$ on $\left(\mathcal{S}^{\prime}, \mathscr{C}, P\right)$, determined by

$$
\mathbb{E}\left[e^{i W^{(\sigma)}(\varphi)}\right]=e^{-\frac{1}{2} \int_{\mathbb{R}}|\widehat{\varphi}(u)|^{2} d \sigma(u)}, \quad \varphi \in \mathcal{S},
$$

satisfies the conditions in Definition 3.1] (and of Lemmas 3.2]3.5).

Definition 3.29. Let $\gamma_{1}$ denote the standard $\mathcal{N}(0,1)$ Gaussian density,

$$
d \gamma_{1}(x)=\frac{1}{\sqrt{2 \pi}} e^{-\frac{x^{2}}{2}} d x \quad \text { on } \mathbb{R}
$$

and set

$$
\Omega_{\gamma}=\times_{\mathbb{N}} \mathbb{R}=\mathbb{R} \times \mathbb{R} \times \cdots,
$$

the infinite Cartesian product, with product measure

$$
Q \stackrel{\text { def }}{=} \times_{\mathbb{N}} \gamma_{1}=\gamma_{1} \times \gamma_{1} \times \cdots
$$

(see [16, 24]), defined on the cylinder sigma-algebra $\mathscr{C}_{\gamma}$ in $\Omega_{\gamma} \stackrel{\text { def }}{=} \times_{\mathbb{N}} \mathbb{R}$.

Specifically, for $f_{n}$ a measurable function on $\mathbb{R}^{n}$, then on $\Omega_{\gamma}$ set $x=\left(x_{1}, x_{2}, \ldots\right)$ with $x_{k} \in \mathbb{R}$ $(k \in \mathbb{N})$,

$$
F(x)=f_{n}\left(x_{1}, \ldots, x_{n}\right),
$$

Then, with this function $f_{n}$ we set:

$$
\begin{aligned}
\int_{\Omega_{\gamma}} F d Q & =\int_{\mathbb{R}^{n}} f_{n}\left(x_{1}, \ldots, x_{n}\right) d \gamma_{1}\left(x_{1}\right) \cdots d \gamma_{1}\left(x_{n}\right) \\
& =\frac{1}{(2 \pi)^{n / 2}} \int_{\mathbb{R}^{n}} f_{n}\left(x_{1}, \ldots, x_{n}\right) e^{-\frac{1}{2} \sum_{k=1}^{n} x_{k}^{2}} d x_{1} \cdots d x_{n} .
\end{aligned}
$$

Remark 3.30. The functions $F$ in (3.31) are called cylinder functions. If the conditions in (3.32) hold for all $n$, then $Q$ is uniquely determined.

\section{Theorem 3.31.}

(i) Every associated Gaussian process $\left(W_{A}^{(\sigma)}\right)_{A \in \mathscr{F}}$ (see Definition 3.1), corresponding to a fixed sigma-finite measure space $(M, \mathscr{F}, \sigma)$ may be realized on $\left(\Omega_{\gamma}, \mathscr{C}_{\gamma}, Q\right)$, i.e. in the canonical infinite Cartesian product measure space of Definition 3.29.

(ii) Given $(M, \mathscr{F}, \sigma)$ and $W^{(\sigma)},(\Omega, \mathscr{C}, P)$ as in Definition [3.1, the realization may be "simulated" by a system of independent, identically distributed (i.i.d.) $\mathcal{N}(0,1)$ random variables $Z_{1}, Z_{2}, \ldots$ If $\left(\varphi_{k}\right)_{k \in \mathbb{N}}$ is an orthonormal basis of real-valued functions in $\mathbf{L}_{2}(M, \mathscr{F}, \sigma)$ then a realization of $W^{(\sigma)}$ in $\left(\Omega_{\gamma}, \mathscr{C}_{\gamma}, Q\right)$ may be written as

$$
W_{A}^{(\sigma)}(\cdot)=\sum_{k=1}^{\infty}\left(\int_{A} \varphi_{k}(x) d \sigma(x)\right) Z_{k}(\cdot),
$$

for all $A \in \mathscr{F}$, where $Z_{k}\left(\left(x_{i}\right)_{i \in \mathbb{N}}\right) \stackrel{\text { def }}{=} x_{k}$ and $\left(x_{i}\right)_{i \in \mathbb{N}} \in \times_{\mathbb{N}} \mathbb{R}$.

Proof. We first note that equation (3.33) is a generalized Karhunen-Loève formula. We proceed to prove that the expression in (3.33) satisfies the condition in Definition 3.1. To 
make sense of (3.33) we shall use the Parseval formula in $\mathbf{L}_{2}(M, \mathscr{F}, \sigma)$. In particular for $A \in \mathscr{F}$ we have

$$
\sigma(A)=\int_{M} \chi_{A}(x) d \sigma(x)=\sum_{k \in \mathbb{N}}\left|\left\langle\chi_{A}, \varphi_{k}\right\rangle_{\sigma}\right|^{2}=\sum_{k \in \mathbb{N}}\left|\int_{A} \varphi_{k}(x) d \sigma(x)\right|^{2} .
$$

It follows that the sum in (3.33) is convergent in $\mathbf{L}_{2}\left(\Omega_{\gamma}, \mathscr{C}_{\gamma}, Q\right)$, and in fact is convergent pointwise a.e. with respect to $Q$. With the use of the dominated convergence theorem we obtain:

$$
\mathbb{E}_{Q}\left[\left|\sum_{k \in \mathbb{N}} \int_{A} \varphi_{k}(x) d \sigma(x) Z_{k}(\cdot)\right|^{2}\right]=\underbrace{\sum_{k \in \mathbb{N}}\left|\int_{A} \varphi_{k}(x) d \sigma(x)\right|^{2}}_{\text {(by }}=\sigma(A),
$$

where we have used

$$
\mathbb{E}_{Q}\left[Z_{n} Z_{m}\right]=\delta_{n, m} \quad \forall n, m \in \mathbb{N} .
$$

Hence the sum representation of $W^{(\sigma)}$ in (3.33) is a well defined random variable belonging to $\mathbf{L}_{2}\left(\Omega_{\gamma}, \mathscr{C}_{\gamma}, Q\right)$. For $A, B \in \mathscr{F}$ we have

$$
\begin{aligned}
\mathbb{E}_{Q}\left[W_{A}^{(\sigma)} W_{B}^{(\sigma)}\right] & =\sum_{k \in \mathbb{N}}\left(\int_{A} \varphi_{k}(x) d \sigma(x)\right)\left(\int_{B} \varphi_{k}(x) d \sigma(x)\right) \\
& =\sum_{k \in \mathbb{N}}\left\langle\chi_{A}, \varphi_{k}\right\rangle_{\sigma}\left\langle\chi_{B}, \varphi_{k}\right\rangle_{\sigma} \\
& =\left\langle\chi_{A}, \chi_{B}\right\rangle_{\sigma} \quad \text { (by Parseval's identity) } \\
& =\sigma(A \cap B),
\end{aligned}
$$

as required in part (2) of Definition 3.1. Verifying the remaining properties in Definition 3.1 is immediate.

Remark 3.32. Note in particular that in representation (3.33) of the random variable $W_{A}^{(\sigma)}$ is independent of the given choice of an orthonormal basis in $\mathbf{L}_{2}(\sigma)$. If $(M, \mathscr{F}, \sigma)=(\mathbb{R}, \mathscr{B}, \lambda)$, with $\lambda$ being the Lebesgue measure on $\mathbb{R}$, then one can take as the underlying orthonormal basis a standard wavelet, for instance the Haar wavelets in $\mathbf{L}_{2}(\mathbb{R})$.

Lemma 3.33. Let $(M, \mathscr{F}, \sigma)$ be a sigma-finite measure space, and let $W^{(\sigma)}$ and $(\Omega, \mathscr{C}, P)$ be an associated Gaussian process, see Definition 3.1 above. Then for every orthonormal basis $\left(\varphi_{k}\right)_{k \in \mathbb{N}}$ in $\mathbf{L}_{2}(M, \mathscr{F}, \sigma)$ the random variables

$$
X_{k}^{(\sigma)}=W^{(\sigma)}\left(\varphi_{k}\right), \quad k \in \mathbb{N}
$$

are a collection of i.i.d. $\mathcal{N}(0,1)$ random variables and

$$
W_{A}^{(\sigma)}(\cdot)=\sum_{k=1}^{\infty}\left(\int_{A} \varphi_{k}(x) d \sigma(x)\right) X_{k}^{(\sigma)}(\cdot) .
$$

Proof. Note that the right side of (3.36) is the Ito integral (see (3.7) in Notation 3.9). Since $W^{(\sigma)}$ is an associated Gaussian process, it follows that $X_{k}^{(\sigma)}$ in (3.36) is Gaussian for all $k \in \mathbb{N}$, with $\mathbb{E}_{P}\left[X_{k}^{(\sigma)}\right]=0$. Moreover, by (3.4) in Lemma 3.5 we have

$$
\mathbb{E}_{P}\left[X_{k}^{(\sigma)} X_{n}^{(\sigma)}\right]=\int_{M} \varphi_{k}(x) \varphi_{n}(x) d \sigma(x)=\left\langle\varphi_{k}, \varphi_{n}\right\rangle_{\sigma}=\delta_{m . n}
$$

since $\left(\varphi_{k}\right)_{k \in \mathbb{N}}$ is an orthonormal basis in $\mathbf{L}_{2}(\sigma)$. We have already proved that the $\left(X_{k}^{(\sigma)}\right)_{k \in \mathbb{N}}$ are Gaussian, so it follows from (3.38) that it is an $\mathcal{N}(0,1)$ i.i.d. collection on $(\Omega, \mathscr{C}, P)$. 
Moreover, by the argument in the proof of Theorem 3.31, we may conclude that representation (3.37) holds, with convergence in $\mathbf{L}_{2}(\Omega, \mathscr{C}, P)$ as well as pointwise $P$ a.e.

Theorem 3.34. Let $(M, \mathscr{F}, \sigma)$ be a sigma-finite measure space and let $\left(W_{A}^{(\sigma)}\right)_{A \in \mathscr{F}}$ be an associated Gaussian process on some probability space $(\Omega, \mathscr{C}, P)$. Let $\left(X_{k}^{(\sigma)}\right)_{k \in \mathbb{N}}$ be the i.i.d. $\mathcal{N}(0,1)$ collection from Lemma 3.33. Set

$$
\Gamma: \Omega \longrightarrow \Omega_{\gamma}=\times_{\mathbb{N}} \mathbb{R},
$$

defined by

$$
\Gamma(\omega)=\left(X_{k}^{(\sigma)}(\omega)\right)_{k \in \mathbb{N}} \in \times_{\mathbb{N}} \mathbb{R}, \quad \omega \in \Omega .
$$

Then $\Gamma$ is measurable and

$$
P\left(\Gamma^{-1}(C)\right)=Q(C)
$$

holds for all $C \in \mathscr{C}_{\gamma}$, i.e. the sigma-algebra of subsets in $\times_{\mathbb{N}} \mathbb{R}$ generated by the cylinder sets.

Remark 3.35. For (3.40), we will use the notation

$$
P \circ \Gamma^{-1}=Q,
$$

with

$$
\Gamma^{-1}(C)=\{\omega \in \Omega \mid \Gamma(\omega) \in C\} .
$$

Proof of Theorem 3.34. Fix $m \in \mathbb{N}$, and let $I_{k}=\left(a_{k}, b_{k}\right], 1 \leq k \leq m$ be a series of finite intervals. It follows from (3.39) that if

$$
C=C_{m}=\left\{\left(x_{k}\right)_{k \in \mathbb{N}} \mid x_{k} \in I_{k}, 1 \leq k \leq m\right\}
$$

then

$$
\Gamma^{-1}(C)=\left\{\omega \in \Omega \mid a_{k} \leq X_{k}^{(\sigma)}(\omega)<b_{k}\right\}
$$

and so $\Gamma$, defined by (3.39), is measurable. But we know that $\left(X_{k}^{(\sigma)}\right)_{k \in \mathbb{N}}$ is an $\mathcal{N}(0,1)$ i.i.d. collection in $(\Omega, \mathscr{C}, P)$, and $\left(Z_{k}\right)_{k \in \mathbb{N}}$ is an $\mathcal{N}(0,1)$ i.i.d. collection in $\left(\Omega_{\gamma}, \mathscr{C}_{\gamma}, Q\right)$. Hence

$$
P\left(\Gamma^{-1}(C)\right)=Q\left(C_{m}\right)=\prod_{k=1}^{m} \gamma_{1}\left(I_{k}\right) .
$$

Thus the desired conclusion (3.40) holds for cylinders in the respective probability spaces. On the other hand, the subset of all sets $C \subset \Omega_{\gamma}$ for which (3.40) holds is a sigma-algebra. It follows that (3.40) is valid on the respective sigma-algebras generated by the cylinder sets.

\subsection{Generalized Fourier transform.}

Corollary 3.36. Let $(M, \mathscr{F}, \sigma)$ be a sigma-finite measure space, and let $\left(W_{A}^{(\sigma)}\right)_{A \in \mathscr{F}}$ be an associated Gaussian process on the probability space $(\Omega, \mathscr{B}, P)$. Then the closed linear span in $\mathbf{L}_{2}(\Omega, \mathscr{B}, P)$ of the functions

$$
\left\{e^{i W_{A}^{(\sigma)}(\cdot)} \mid A \in \mathscr{F}\right\}
$$

is $\mathbf{L}_{2}(\Omega, \mathscr{B}, P)$.

Proof. By Theorem 3.34 we may assume that $\left(W_{A}^{(\sigma)}\right)_{A \in \mathscr{F}}$ is realized in $\mathbf{L}_{2}\left(\times_{\mathbb{N}} \mathbb{R}, \mathscr{C}_{\gamma}, Q\right)$, with $Q=\times_{\mathbb{N}} \gamma_{1}$ being the infinite dimensional measure on $\Omega_{\gamma}=\times_{\mathbb{N}} \mathbb{R}$; see also Theorem 3.31, But in this infinite-product space the conclusion is clear. If $F \in \mathbf{L}_{2}\left(\times_{\mathbb{N}} \mathbb{R}, \mathscr{C}_{\gamma}, Q\right)$ satisfies

$$
\int_{\times_{\mathbb{N}} \mathbb{R}} F e^{i W_{A}} d Q=0, \quad \forall A \in \mathscr{F},
$$


then a direct computation, using (3.33), shows that $F=0$ a.e. $Q$.

Definition 3.37. Let $(M, \mathscr{F}, \sigma)$ be a sigma-finite measure space, and consider the positive definite function

$$
K^{(\sigma)}(A, B)=e^{-\frac{\sigma(A)+\sigma(B)}{2}} e^{\sigma(A \cap B)}=e^{-\frac{1}{2}\left\|\chi_{A}-\chi_{B}\right\|_{\sigma}^{2}}, \quad A, B \in \mathscr{F} .
$$

We shall denote the corresponding reproducing kernel Hilbert space by $\mathcal{H}^{(\sigma)}$.

Corollary 3.38. (The generalized Fourier transform.) Let $\left(W_{A}^{(\sigma)}\right)_{A \in \mathscr{F}}$ be a Gaussian process associated with the sigma-finite measure space $(M, \mathscr{F}, \sigma)$, and let $(\Omega, \mathscr{B}, P)$ be the underlying probability space. Let $\mathcal{H}^{(\sigma)}$ be the reproducing kernel Hilbert space as in Definition 3.37. For $F \in \mathbf{L}_{2}(\Omega, \mathscr{B}, P)$, set

$$
\widehat{F}(A)=\mathbb{E}_{P}\left[F e^{i W_{A}}\right], \quad A \in \mathscr{F} .
$$

Then the map $F \mapsto \widehat{F}$ is unitary from $\mathbf{L}_{2}(\Omega, \mathscr{B}, P)$ onto $\mathcal{H}^{(\sigma)}$.

Proof. Using Corollary 3.36, it is enough to prove that for all $A, B \in \mathscr{F}$ we have

$$
\mathbb{E}\left[e^{-i W_{B}} e^{i W_{A}}\right]=K^{(\sigma)}(A, B)=e^{-\left\{\frac{\sigma(A)+\sigma(B)}{2}+\sigma(A \cap B)\right\}}
$$

(see (3.43) $)$. A direct computation yields

$$
\begin{aligned}
E\left[e^{-i W_{B}} e^{i W_{A}}\right] & =E\left[e^{i\left(W_{A}-W_{B}\right)}\right] \\
& =e^{-\frac{1}{2}\left\|\chi_{A}-\chi_{B}\right\|_{\sigma}^{2}} \quad(\text { by Theorem 3.34) } \\
& =K^{(\sigma)}(A, B) \quad(\text { by }(3.43))
\end{aligned}
$$

for all $A, B \in \mathscr{F}$.

Corollary 3.39. Let $\left(W_{A}^{(\sigma)}\right)_{A \in \mathscr{F}}$ be a collection of Gaussian random variables associated with a sigma-finite measure space $(M, \mathscr{F}, \sigma)$. Let $\left(\varphi_{k}\right)_{k \in \mathbb{N}}$ be an orthonormal basis of $\mathbf{L}_{2}(M, \mathscr{F}, \sigma)$ (we denote this space by $\mathbf{L}_{2}(\sigma)$ ), and let $\Gamma: \Omega \longrightarrow \times_{\mathbb{N}} \mathbb{R} \stackrel{\text { def. }}{=} \Omega_{\gamma}$, with

$$
\Gamma(\omega)=\left(X_{k}^{(\sigma)}(\omega)\right)_{k \in \mathbb{N}} .
$$

being the corresponding coordinate system, i.e.

$$
X_{k}^{(\sigma) \stackrel{\text { def. }}{=}} W^{(\sigma)}\left(\varphi_{k}\right)=\int_{M} \varphi_{k}(x) d W^{(\sigma)}(x)
$$

being the Ito-integral representation of (3.7). Then

$$
\Gamma\left(\mathbf{L}_{2}(\sigma)\right) \subset \ell^{2} \subset \times_{\mathbb{N}} \mathbb{R}
$$

Proof. Recall that we consider real-valued functions. Let $\langle\cdot, \cdot\rangle_{\sigma}$ denote the standard inner product in $\mathbf{L}_{2}(\sigma)$. Then for all $f \in \mathbf{L}_{2}(\sigma)$ we have

$$
W^{(\sigma)}(f) \in \mathbf{L}_{2}(\Omega, P),
$$

and, by Lemma 3.5.

$$
\Gamma(f)=\left(\left\langle f, \varphi_{k}\right\rangle_{\sigma}\right)_{k \in \mathbb{N}} \in \ell_{2} \subset \times_{\mathbb{N}} \mathbb{R}
$$

and

$$
\mathbb{E}_{P}\left[\left(W^{(\sigma)}(f)\right)^{2}\right]=\|f\|_{\sigma}^{2}=\sum_{k \in \mathbb{N}}\left|\left\langle f, \varphi_{k}\right\rangle\right|^{2},
$$


and

$$
W^{(\sigma)}(f)=\sum_{k \in \mathbb{N}}\left\langle f, \varphi_{k}\right\rangle_{k} X_{k}^{(\sigma)}
$$

is well defined, Gaussian, and satisfies (3.48).

Theorem 3.40. Let $(M, \mathscr{F}, \sigma),\left(W_{A}^{(\sigma)}\right)_{A \in \mathscr{F}}$ and $(\Omega, \mathscr{B}, P)$ be as in Corollary 3.39. Then the measure $P$ is quasi-invariant with respect to the $\mathbf{L}_{2}(\sigma)$ translations in $\Omega$. More precisely, we have

$$
\int_{\Omega} F(\cdot+f) d P(\cdot)=\int_{\Omega} F(\cdot) e^{-\frac{1}{2}\|f\|_{\sigma}^{2}+W^{(\sigma)}(f)(\cdot)} d P(\cdot)
$$

for all $F \in \mathbf{L}_{2}(\Omega, P)$ and all $f \in \mathbf{L}_{2}(\sigma)$.

Proof. Note that in the formulation of (3.50) we make use of the coordinate system

$$
\Gamma: \Omega \longrightarrow \times_{\mathbb{N}} \mathbb{R}
$$

from Theorem 3.34 and Corollary 3.39 . Hence the term $e^{-\frac{1}{2}\|f\|_{\sigma}^{2}+W^{(\sigma)}(f)(\cdot)}$ is the associated Radon-Nikodym derivative. Using Theorem 3.34, and passing to cylinder functions, we note that (3.50) follows from a computation of $\mathbb{R}^{m}$-integrals for all $m \in \mathbb{N}$. We check that if $F_{m}$ is $\mathbf{L}_{2}$ with respect to the standard $\mathbb{R}^{m}$-Gaussian joint density

$$
\gamma_{m}=\underbrace{\gamma_{1} \times \cdots \times \gamma_{1}}_{m \text { times }}
$$

then

$$
\begin{aligned}
\iint_{\mathbb{R}^{m}} F_{m}\left(x_{1}+\left\langle f, \varphi_{1}\right\rangle_{\sigma}, \ldots, x_{m}+\left\langle f, \varphi_{m}\right\rangle_{\sigma}\right) d \gamma_{m}\left(x_{1}, \ldots, x_{m}\right)= \\
=\iint_{\mathbb{R}^{m}} F_{m}\left(x_{1}, \ldots, x_{m}\right) e^{-\frac{1}{2} \sum_{k=1}^{m}\left\langle f, \varphi_{k}\right\rangle_{\sigma}^{2}} e^{\sum_{k=1}^{m} x_{k}\left\langle f, \varphi_{k}\right\rangle_{\sigma}} d \gamma_{m}\left(x_{1}, \ldots, x_{m}\right) .
\end{aligned}
$$

From Theorem 3.34 and Corollary 3.39 we have that

$$
W^{(\sigma)}(f)(\cdot)=\sum_{k=1}^{\infty}\left\langle f, \varphi_{k}\right\rangle_{\sigma} X_{k}^{(\sigma)}(\cdot)
$$

and

$$
\mathbb{E}_{\sigma}\left[\left(W^{(\sigma)}(f)\right)^{2}\right]=\sum_{k=1}^{m}\left\langle f, \varphi_{k}\right\rangle_{\sigma}^{2}=\|f\|_{\mathbf{L}_{2}(\sigma)}^{2} .
$$

Using again Theorem 3.34 and Kolmogorov's induction limit construction, we finally note that the desired formula (3.50) follows from (3.51)-(3.53) above.

Our quadratic variation result in the first part of the present section is motivated by, and is a generalization of a classical theorem for the Brownian motion, often called Lévy's theorem, see e.g. 9. Similarly, our decomposition theorem (Theorem 3.31) is motivated by, and extends a classical result often called a Kahrunen-Loève decomposition, see e.g. [11]. In the above, we have made use of the theory of Ito-integration and its generalizations; see e.g. [23, 46, 19, 18, 21, 17.

\section{Gaussian stochastic calculus}

In this section, a preparation for Section 5, we return to the Gaussian process of Section 2 , applying results of Section 3 . 
4.1. Gaussian processes and tempered measures: The Minlos theorem. Let $\sigma$ be a tempered measure. The map

$$
\varphi \mapsto e^{-\frac{1}{2} \int_{\mathbb{R}}|\widehat{\varphi}(u)|^{2} d \sigma(u)}
$$

is continuous and positive definite on $\mathcal{S}$ (see [2, Proposition 3.3, p. 714]). An application of Minlos' theorem (see [13]) yields:

Theorem 4.1. For every tempered measure $\sigma$ there exists a uniquely defined probability measure $P_{\sigma}$ on $\mathcal{S}^{\prime}$, defined on the sigma-algebra $\mathscr{C}=\mathscr{C}\left(\mathcal{S}^{\prime}\right)$ generated by the cylinder sets (see (2.4) ), and determined by the following condition:

$$
\mathbb{E}_{P_{\sigma}}\left[e^{i X_{\varphi}^{(\sigma)}}\right]=\int_{\mathcal{S}^{\prime}} e^{i\langle\varphi, \xi\rangle} d P_{\sigma}(\xi)=e^{-\frac{1}{2} \int_{\mathbb{R}}|\widehat{\varphi}(u)|^{2} d \sigma(u)} .
$$

Using the corresponding expectation

$$
\mathbb{E}_{\sigma}[U]=\int_{\mathcal{S}^{\prime}} U(\omega) P_{\sigma}(\omega)
$$

(4.2) takes the equivalent form

$$
\mathbb{E}_{\sigma}\left[e^{i\langle\cdot, \varphi\rangle}\right]=e^{-\frac{1}{2}\|\widehat{\varphi}\|_{\sigma}^{2}} .
$$

In the above we make use of reproducing kernel Hilbert spaces and their association with the study of Gaussian processes; see e.g. [45, 30, 15, 12, 48].

\subsection{A Gaussian process realized on $\mathcal{S}^{\prime}$.}

Lemma 4.2. The pairing (2.1) between $\mathcal{S}$ and $\mathcal{S}^{\prime}$ extends to a pairing between $\mathbf{L}_{2}(\mathbb{R})$ and $\mathcal{S}^{\prime}$. The stochastic process

$$
X_{\varphi}^{(\sigma)}(\xi)=\langle\xi, \varphi\rangle, \quad \varphi \in \mathcal{S}
$$

may be extended to a process

$$
X_{f}^{(\sigma)}(\xi)=\langle\xi, f\rangle, \quad f \in \mathbf{L}_{2}(\mathbb{R}, d x) .
$$

Proof. Set

$$
X_{t}^{(\sigma)}(\xi)=\left\langle\xi, \chi_{[0, t]}\right\rangle
$$

$X_{t}^{(\sigma)}$ is a zero mean Gaussian process with variance

$$
\int_{\mathbb{R}}|\widehat{\chi[0, t]}(u)|^{2} d \sigma(u)=2 \int_{\mathbb{R}} \frac{1-\cos (u t)}{u^{2}} d \sigma(u)=4 \int_{\mathbb{R}} \frac{\sin ^{2}\left(\frac{u t}{2}\right)}{u^{2}} d \sigma(u) .
$$

In the following lemma we compute the covariance and related quantities. In formulas (4.7) and (4.8) below we set

$$
r_{\sigma}(t)=\mathbb{E}_{\sigma}\left[\left|X_{t}^{(\sigma)}\right|^{2}\right]
$$


Lemma 4.3. We set $X_{0}^{(\sigma)}=0$. The following formulas hold:

$$
\begin{aligned}
\mathbb{E}_{\sigma}\left[\left|X_{t}^{(\sigma)}\right|^{2}\right] & =4 \int_{\mathbb{R}} \frac{\sin ^{2}\left(\frac{u t}{2}\right)}{u^{2}} d \sigma(u), \\
\mathbb{E}_{\sigma}\left[\left|X_{t}^{(\sigma)}-X_{s}^{(\sigma)}\right|^{2}\right] & =r_{\sigma}(s-t) \\
\mathbb{E}_{\sigma}\left[X_{t}^{(\sigma)} X_{s}^{(\sigma)}\right] & =\frac{r_{\sigma}(t)+r_{\sigma}(s)-r_{\sigma}(s-t)}{2} .
\end{aligned}
$$

The computations are standard and will be omitted.

Thus $X^{(\sigma)}$ is a stationary-increment Gaussian process. Recall that these are (not necessarily real) Gaussian processes with covariance function of the form

$$
2 \mathbb{E}_{\sigma}\left[X_{t}^{(\sigma)} X_{s}^{(\sigma)}\right]=r(t)+\overline{r(s)}-r(t-s), \quad t, s \in \mathbb{R},
$$

Functions $r$ for which the kernel (4.9) is positive definite on the real line have been investigated by Schoenberg, von Neumann and Krein; see [36, 28]. They are of the form

$$
r(t)=r_{0}+i c t-\int_{\mathbb{R}}\left\{e^{i t v}-1-\frac{i t v}{v^{2}+1}\right\} d \sigma(v)
$$

where $r_{0} \geq 0, c \in \mathbb{R}$ and $\sigma$ is a tempered measure satisfying (2.2) for $p=1$. In the case where $\sigma$ is even, one recovers formula (4.4).

We note that such processes admit derivatives which are generalized stationary processes with covariance $\widehat{\sigma}(t-s)$, with the Fourier transform being computed in the sense of distributions. See [1, 3, 2] for more information.

\section{A Gaussian process realized on $C(\mathbb{R})$.}

We are now ready to introduce the two realizations of probability spaces for the family of Gaussian processes considered above.

Theorem 5.1. There is a unique measure $Q_{\sigma}$ defined on the cylinder sigma-algebra of $\Omega=$ $C(\mathbb{R})$ such that

$$
X_{t}^{(\sigma)}(\omega)=\omega(t), \quad t \in \mathbb{R}, \quad \omega \in \Omega=C(\mathbb{R}),
$$

and $X_{0}^{(\sigma)}=0$ is a Gaussian process with zero mean and covariance

$$
\mathbb{E}_{Q_{\sigma}}\left[X_{t}^{(\sigma)} X_{s}^{(\sigma)}\right]=\frac{r_{\sigma}(t)+r_{\sigma}(s)-r_{\sigma}(s-t)}{2}
$$

(see (4.8)), with $r_{\sigma}$ as in (4.5).

Proof. For a finite set $t_{1}, \ldots, t_{n} \in \mathbb{R}$ of sample points and a measurable function $f_{n}$ on $\mathbb{R}^{n}$ we set

$$
F(\omega)=f_{n}\left(\omega\left(t_{1}\right), \ldots, \omega\left(t_{n}\right)\right), \quad \omega \in \mathbb{C}(R) .
$$

For $a>0$ define

$$
g_{a}(x)=\frac{1}{a \sqrt{2 \pi}} e^{-\frac{x^{2}}{2 a^{2}}}, \quad x \in \mathbb{R} .
$$


and for $0<t_{1}<t_{2}<\ldots<t_{n}$ let

$$
\begin{aligned}
\mathcal{L}_{n}(F) & =\int_{\mathbb{R}^{n}} f_{n}\left(x_{1}, \ldots, x_{n}\right) g_{r_{\sigma}\left(t_{1}\right)}\left(x_{1}\right) g_{r_{\sigma}\left(t_{2}-t_{1}\right)}\left(x_{2}-x_{1}\right) \cdots g_{r_{\sigma}\left(t_{n}-t_{n-1}\right)}\left(x_{n}-x_{n-1}\right) d x_{1} d x_{2} \cdots d x_{n} \\
& =\int_{\mathbb{R}^{n}} f_{n}\left(x_{1}, x_{2}+x_{1}, \ldots, x_{n}+x_{n-1}+\cdots+x_{1}\right) g_{r_{\sigma}\left(t_{1}\right)}\left(x_{1}\right) g_{r_{\sigma}\left(t_{2}-t_{1}\right)}\left(x_{2}\right) \cdots g_{r_{\sigma}\left(t_{n}-t_{n-1}\right)}\left(x_{n}\right) d x_{1} d x_{2} \cdots d x_{n}
\end{aligned}
$$

where $f_{n}$ is the function in $\mathbb{R}^{n}$ introduced in (5.3).

Using Kolmogorov's theorem we see that there is a unique measure $Q_{\sigma}$ with the property stated in the theorem such that for a cylinder function $F$ as in (5.3) we have

$$
\int_{\Omega} F d Q_{\sigma}=\mathcal{L}_{n}(F)
$$

The other claims are easily verified. We omit the details.

In this section we make use of the two general procedures for constructing Gaussian processes, and the corresponding probability spaces. To simplify matters, we divide them into $(i)$ the inductive limit construction first proposed by Kolmogorov, and (ii) the alternative approach based on Gelfand triples. For references to the first, see [27, 14, for the second, see e.g., [29].

We further mention the following result from [2] and [3] (we refer the reader to these references for the proof).

Theorem 5.2. Let $\sigma$ be a tempered measure (see Definition 2.1) and let $\left(X_{\varphi}^{(\sigma)}\right)_{\varphi \in \mathcal{S}}$ be the corresponding Gaussian process indexed by the real $S c h w a r t z$ space $\mathcal{S}$. Further, let $P_{W}$ denote the standard Wiener-measure. For $f \in \mathbf{L}_{2}(\mathbb{R})$, let $\tilde{f}$ denote the corresponding Gaussian process with zero mean and covariance

$$
\mathbb{E}_{P_{W}}\left(|\widetilde{f}|^{2}\right)=\|f\|_{2}^{2} .
$$

Then, there exists a continuous linear operator $Q^{(\sigma)}$ from $\mathcal{S}$ into $\mathbf{L}_{2}(\mathbb{R})$ such that

$$
\mathbb{E}_{P_{W}}\left[e^{i Q^{(\sigma)}(\varphi)}\right]=\mathbb{E}_{P_{\sigma}}\left[e^{i X_{\varphi}^{(\sigma)}}\right]=e^{-\frac{1}{2} \int_{\mathbb{R}}|\widehat{\varphi}(u)|^{2} d \sigma(u)} .
$$

\section{Equivalence of measures}

In this section we provide details (Theorem 6.4 below) associated with the equivalence of the two realizations of probability spaces for the family of Gaussian processes considered above.

6.1. Two probability spaces. In the approach based on Gelfand triples we realize the family of stationary-increment processes on the probability space $\left(\mathcal{S}^{\prime}, \mathscr{C}\left(\mathcal{S}^{\prime}\right), P_{\sigma}\right)$, while in the approach based instead on Kolmogorov consistency theorem, a probability space is $\left(C(\mathbb{R}), \mathscr{C}, Q_{\sigma}\right)$, where $\mathscr{C}$ is the corresponding cylinder sigma-algebra. In both cases the Gaussian process is indexed by a fixed tempered measure $\sigma$.

The setting in the present section is as follows. Fix a tempered measure $\sigma$ on $\mathbb{R}$ and consider the above two variations of the $\sigma$-Gaussian process, For $\omega \in C(\mathbb{R})$ let $\omega^{\prime}$ be the corresponding Schwartz tempered distribution.

We shall write $X_{\text {Kolm }}^{(\sigma)}(t)$ for the process in the Kolmogorov realization, and $X_{\text {Gel }}^{(\sigma)}$ for the Gelfand-triple realization, i.e.

$$
\begin{aligned}
X_{\mathrm{Kolm}}^{(\sigma)}(t)(\omega) & =\omega(t), \quad \forall \omega \in C(\mathbb{R}), \forall t \in \mathbb{R}, \\
X_{\mathrm{Gel}}^{(\sigma)}(t)(\xi) & =\left\langle\xi, \chi_{[0, t]}\right\rangle, \quad \forall \xi \in \mathcal{S}^{\prime}, \forall t \in \mathbb{R} .
\end{aligned}
$$


6.2. Equivalence. In the following statement recall that cylinder sets have been defined in (2.4).

Lemma 6.1. Let $\sigma$ be a tempered measure as introduced in Section 4 (see Lemmas 4.2 and 4.3). Then the Gaussian process $\left(X_{\varphi}^{(\sigma)}\right)_{\varphi \in \mathcal{S}}$ determined by

$$
\mathbb{E}\left[X_{\varphi}^{(\sigma)}\right]=0 \quad \text { and } \quad \mathbb{E}\left[e^{i X_{\varphi}^{(\sigma)}}\right]=e^{-\frac{1}{2} \int_{\mathbb{R}}|\widehat{\varphi}(u)|^{2} d \sigma(u)}
$$

extends from $\mathcal{S}$ to $\mathbf{L}_{2}(\mathbb{R})$, i.e. the $\mathcal{S}-\mathcal{S}^{\prime}$ pairing $X_{\varphi}^{(\sigma)}(\xi)=\langle\xi, \varphi\rangle$ extends uniquely to $X_{f}^{(\sigma)}$. By abuse of notation we shall write

$$
X_{f}^{(\sigma)}(\xi)=\langle\xi, f\rangle
$$

Proof. For $f \in \mathbf{L}_{2}(\mathbb{R})$, interpret $X_{f}^{(\sigma)}(\xi)$ as the Ito-integral

$$
X_{f}^{(\sigma)}(\xi)=\int_{\mathbb{R}} f(x) d X^{(\sigma)}(x),
$$

where the right hand side of (6.3) is computed with the use of Lemma 3.5.

Fix $k \in \mathbb{N}$, and consider a Gaussian measure $Q$ on $C\left(\mathbb{R}^{k}\right)$ constructed from Gaussian transition probabilities, see e.g. (5.4)-(5.3) above. We denote by

$$
C_{\text {temp }}\left(\mathbb{R}^{k}\right)=C\left(\mathbb{R}^{k}\right) \cap \mathcal{S}_{\mathbb{R}^{k}}^{\prime}
$$

the set of tempered continuous functions on $\mathbb{R}^{k}$ (see [47]). Furthermore, let $\mathbb{R}^{k}=\left(\mathbb{R}^{k} \cup\{\infty\}\right)^{\sim}$ denote the one-point compactification of $\mathbb{R}^{k}$. Then we have:

Lemma 6.2. It holds that

$$
C\left(\mathbb{R}^{k}\right) \cap \prod_{\mathbb{R}^{k}} \stackrel{\mathbb{R}}{k}^{k} \subset C_{\text {temp }}\left(\mathbb{R}^{k}\right) .
$$

Proof. The functions $\omega$ in the intersection on the left hand side of (6.4) have well defined limits at $\infty$. There are in particular bounded and hence tempered.

Lemma 6.3. The Gaussian measure $Q_{\sigma}$ in Theorem 5.1 is supported in $C\left(\mathbb{R}^{k}\right) \cap \prod_{\mathbb{R}^{k}} \mathbb{R}^{k}$

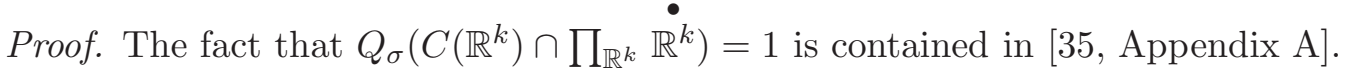

Theorem 6.4. For a fixed tempered measure $\sigma$ let $X_{\mathrm{Kolm}}^{(\sigma)}$ and $X_{\mathrm{Gel}}^{(\sigma)}$ be the associated Gaussian processes arising from Kolmogorov and Gelfand constructions respectively. Let

$$
T: C_{\text {temp }}(\mathbb{R}) \longrightarrow \mathcal{S}^{\prime}
$$

be defined by

$$
T(\omega)=\omega^{\prime}, \quad \omega \in C_{\text {temp }}(\mathbb{R}) ;
$$

(the prime in $\omega^{\prime}$ denotes the derivative in the sense of distributions). Then,

$$
X_{\mathrm{Gel}}^{(\sigma)}\left(\omega^{\prime}\right)=X_{\mathrm{Kolm}}^{(\sigma)}(\omega)=\omega(t),
$$

and

$$
Q_{\sigma} \circ T^{-1}=P_{\sigma}
$$

meaning that for all cylinder sets $\Delta \subset \mathcal{S}^{\prime}$, with

$$
T^{-1}(\Delta)=\left\{\omega \in C_{\text {temp }}(\mathbb{R}) \text { such that } \omega^{\prime} \in \Delta\right\},
$$

then

$$
Q_{\sigma}\left(T^{-1}(\Delta)\right)=P_{\sigma}(\Delta)
$$


Proof. To prove (6.6) let $\omega \in C_{\text {temp }}(\mathbb{R})$ and $t \in \mathbb{R}$. Since the process is assumed to be zero at $t=0$ we can write

$$
\begin{aligned}
X_{\mathrm{Gel}}^{(\sigma)}\left(\omega^{\prime}\right) & =\left\langle\omega^{\prime}, \chi_{[0, t]}\right\rangle \\
& =-\left\langle\chi_{[0, t]}^{\prime}, \omega\right\rangle \\
& =\omega(t) \\
& =X_{\text {Kolm }}^{(\sigma)}(t)(\omega) .
\end{aligned}
$$

We now turn to the proof of (6.7). Note that this yields an explicit formula for the measure $P_{\sigma}$ on $\mathcal{S}^{\prime}$ which was previously obtained indirectly as an application of Minlos' theorem to the right hand side of (3.6), i.e. the Minlos-Gelfand approach does not yield a construction of $P_{\sigma}$, only existence. Let $A \subset \mathbb{R}^{n}$ be a Borel set. Consider a cylinder set of the form (2.4), with

Then,

$$
\varphi_{k}(u)=\chi_{\left[0, t_{k}\right]}(u), \quad k=1, \ldots, n
$$

$P_{\sigma}\left\{\omega^{\prime} \in \mathcal{S}^{\prime} \mid\left(\left\langle\omega^{\prime}, \chi_{\left[0, t_{1}\right]}\right\rangle, \ldots,\left\langle\omega^{\prime}, \chi_{\left[0, t_{n}\right]}\right\rangle\right) \in A\right\}=Q_{\sigma}\left\{\omega \in C_{\text {temp }}(\mathbb{R}) \mid\left(\omega\left(t_{1}\right), \ldots, \omega\left(t_{n}\right)\right) \in A\right\}$,

where $P_{\sigma}$ and $Q_{\sigma}$ refer to the functional measures on $\mathcal{S}^{\prime}$ and $C(\mathbb{R})$ respectively.

Acknowledgment: It is a pleasure to thank the referee for his comments on the first version of the paper.

\section{REFERENCES}

[1] D. Alpay, H. Attia, and D. Levanony. On the characteristics of a class of Gaussian processes within the white noise space setting. Stochastic processes and applications, 120:1074-1104, 2010.

[2] D. Alpay and P. Jorgensen. Stochastic procesees induced by singular operators. Numerical Functional Analysis and Optimization, 33:708-735, 2012.

[3] D. Alpay, P. Jorgensen, and D. Levanony. A class of Gaussian processes with fractional spectral measures. J. Funct. Anal., 261(2):507-541, 2011.

[4] M. A. Al'shanskiı̌. Gaussian white noise with trajectories in the space $\mathscr{S}^{\prime}(H)$. Izv. Vyssh. Uchebn. Zaved. Mat., (5):3-11, 2011.

[5] K. Anjyo and J. P. Lewis. RBF interpolation and Gaussian process regression through an RKHS formulation. J. Math-for-Ind., 3A:63-71, 2011.

[6] D. Applebaum. Lévy processes and stochastic calculus, volume 116 of Cambridge Studies in Advanced Mathematics. Cambridge University Press, Cambridge, second edition, 2009.

[7] D. Applebaum. Corrigendum: Probability measures on compact groups which have square-integrable densities [mr2471953]. Bull. Lond. Math. Soc., 42(5):948, 2010.

[8] D. Applebaum. Infinitely divisible central probability measures on compact Lie groups - regularity, semigroups and transition kernels. Ann. Probab., 39(6):2474-2496, 2011.

[9] H. Bühlmann. $L^{2}$-martingales and orthogonal decomposition. Z. Wahrscheinlichkeitstheorie und Verw. Gebiete, 1:394-414, 1962/1963.

[10] T.E. Duncan and B. Pasik-Duncan. Linear-exponential-quadratic Gaussian control for stochastic equations in a Hilbert space. Dynam. Systems Appl., 21(2-3):407-416, 2012.

[11] A. Dür. On the optimality of the discrete Karhunen-Loève expansion. SIAM J. Control Optim., 36(6):1937-1939 (electronic), 1998.

[12] M. Erraoui and E. H. Essaky. Canonical representation for Gaussian processes. In Séminaire de Probabilités XLII, volume 1979 of Lecture Notes in Math., pages 365-381. Springer, Berlin, 2009.

[13] I. M. Gel'fand and N. Ya. Vilenkin. Generalized functions. Vol. 4. Academic Press [Harcourt Brace Jovanovich Publishers], New York, 1964 [1977]. Applications of harmonic analysis, Translated from the Russian by Amiel Feinstein.

[14] M. Grothaus, Y.G. Kondratiev, and L. Streit. Regular generalized functions in Gaussian analysis. Infin. Dimens. Anal. Quantum Probab. Relat. Top., 2(1):1-25, 1999.

[15] T. Heinosaari, A.S. Holevo, and M. Wolf. The semigroup structure of Gaussian channels. Quantum Inf. Comput., 10(7-8):619-635, 2010. 
[16] T. Hida. Brownian motion, volume 11 of Applications of Mathematics. Springer-Verlag, New York, 1980. Translated from the Japanese by the author and T. P. Speed.

[17] T. Hida. Some of the recent topics on stochastic analysis. In Stochastic analysis and applications, volume 2 of Abel Symp., pages 383-397. Springer, Berlin, 2007.

[18] T. Hida. Perspectives of white noise analysis. In Quantum bio-informatics, volume 21 of $Q P-P Q: Q u a n t u m$ Probab. White Noise Anal., pages 111-125. World Sci. Publ., Hackensack, NJ, 2008.

[19] T. Hida. Scientific works by Wiener and Lévy after Brown. In Collective dynamics: topics on competition and cooperation in the biosciences, volume 1028 of AIP Conf. Proc., pages 1-10. Amer. Inst. Phys., Melville, NY, 2008.

[20] T. Hida and M. Hitsuda. Gaussian processes, volume 120 of Translations of Mathematical Monographs. American Mathematical Society, Providence, RI, 1993. Translated from the 1976 Japanese original by the authors.

[21] T. Hida and Si Si. Lectures on white noise functionals. World Scientific Publishing Co. Pte. Ltd., Hackensack, NJ, 2008.

[22] A. Holevo. Probabilistic and statistical aspects of quantum theory, volume 1 of Quaderni. Monographs. Edizioni della Normale, Pisa, second edition, 2011. With a foreword from the second Russian edition by K. A. Valiev.

[23] K. Itô. Stochastic differentials. Appl. Math. Optim., 1(4):374-381, 1974/75.

[24] S. Kakutani. Spectral analysis of stationary Gaussian processes. In Proc. 4th Berkeley Sympos. Math. Statist. and Prob., Vol. II, pages 239-247. Univ. California Press, Berkeley, Calif., 1961.

[25] S. Khachatryan, R. Schrader, and A. Sedrakyan. Grassmann-Gaussian integrals and generalized star products. J. Phys. A, 42(30):304019, 16, 2009.

[26] A. S. Kholevo and M. E. Shirokov. Mutual and coherent information for infinite-dimensional quantum channels. Problemy Peredachi Informatsii, 46(3):3-21, 2010.

[27] A. N. Kolmogorov. Foundations of the Theory of Probability. Chelsea Publishing Company, New York, N. Y., 1950.

[28] M.G. Krein. On the problem of continuation of helical arcs in Hilbert space. C. R. (Doklady) Acad. Sci. URSS (N.S.), 45:139-142, 1944.

[29] I. Kubo, H.-H. Kuo, and A. Sengupta. White noise analysis on a new space of Hida distributions. Infin. Dimens. Anal. Quantum Probab. Relat. Top., 2(3):315-335, 1999.

[30] Th. Kühn. Covering numbers of Gaussian reproducing kernel Hilbert spaces. J. Complexity, 27(5):489499, 2011.

[31] M.A. Lifshits. Gaussian random functions, volume 322 of Mathematics and its Applications. Kluwer Academic Publisher, 1995.

[32] J.-L. Lions. Remarks on reproducing kernels of some function spaces. In Function spaces, interpolation theory and related topics (Lund, 2000), pages 49-59. de Gruyter, Berlin, 2002.

[33] M. Loève. Probability theory. II. Springer-Verlag, New York, fourth edition, 1978. Graduate Texts in Mathematics, Vol. 46.

[34] S. V. Lototsky and K. Stemmann. Stochastic integrals and evolution equations with Gaussian random fields. Appl. Math. Optim., 59(2):203-232, 2009.

[35] E. Nelson. Feynman integrals and the Schrödinger equation. J. Mathematical Phys., 5:332-343, 1964.

[36] J. von Neumann and I. J. Schoenberg. Fourier integrals and metric geometry. Trans. Amer. Math. Soc., 50:226-251, 1941.

[37] I. Nourdin and G. Peccati. Normal approximations with Malliavin calculus, volume 192 of Cambridge Tracts in Mathematics. Cambridge University Press, Cambridge, 2012. From Stein's method to universality.

[38] K. R. Parthasarathy. What is a Gaussian state? Commun. Stoch. Anal., 4(2):143-160, 2010.

[39] K. R. Parthasarathy and K. Schmidt. Positive definite kernels, continuous tensor products, and central limit theorems of probability theory. Springer-Verlag, Berlin, 1972. Lecture Notes in Mathematics, Vol. 272.

[40] K. R. Parthasarathy and K. Schmidt. Stable positive definite functions. Trans. Amer. Math. Soc., 203:161$174,1975$.

[41] D. Revuz and M. Yor. Continuous Martingales and Brownian Motion. Springer, 1991.

[42] L.E.C. Rosales-Zárate and P.D. Drummond. Resolution of unity for fermionic Gaussian operators. J. Phys. A, 46(27):275203, 16, 2013.

[43] G. V. Ryabov. Finite absolute continuity of Gaussian measures on infinite-dimensional spaces. Ukraïn. Mat. Zh., 60(10):1367-1377, 2008.

[44] R. Schrader, Hermann Schulz-Baldes, and Ara Sedrakyan. Perturbative test of single parameter scaling for 1D random media. Ann. Henri Poincaré, 5(6):1159-1180, 2004. 
[45] N. Srinivas, A. Krause, Sham M. Kakade, and M.W. Seeger. Information-theoretic regret bounds for Gaussian process optimization in the bandit setting. IEEE Trans. Inform. Theory, 58(5):3250-3265, 2012.

[46] V. Tesko. The integration of operator-valued functions with respect to vector-valued measures. Methods Funct. Anal. Topology, 18(3):288-304, 2012.

[47] F. Treves. Topological vector spaces, distributions and kernels. Academic Press, 1967.

[48] A. W. van der Vaart and J. H. van Zanten. Reproducing kernel Hilbert spaces of Gaussian priors. In Pushing the limits of contemporary statistics: contributions in honor of Jayanta K. Ghosh, volume 3 of Inst. Math. Stat. Collect., pages 200-222. Inst. Math. Statist., Beachwood, OH, 2008.

(DA) Department of Mathematics, Ben-Gurion University of the Negev, Beer-Sheva 84105, IsRAEL

E-mail address: dany@math.bgu.ac.il

(PJ) Department of Mathematics, University of Iowa. Iowa City, IA 52242 USA

E-mail address: palle-jorgensen@uiowa.edu

(DL) Department of Electrical Engineering, Ben Gurion University of the Negev,, Beer Sheva 84105, ISRAEL

E-mail address: levanony@ee.bgu.ac.il 